\title{
Crossflow instability of finite Bödewadt flows: Transients and spiral waves
}

\author{
Juan M. Lopez, ${ }^{1, a)}$ Francisco Marques, ${ }^{2}$ Antonio M. Rubio, ${ }^{1}$ and Marc Avila ${ }^{3}$ \\ ${ }^{1}$ School of Mathematical and Statistical Sciences, Arizona State University, Tempe, Arizona 85287, USA \\ ${ }_{3}^{2}$ Departament de Física Aplicada, Universitat Politècnica de Catalunya, 08034 Barcelona, Spain \\ ${ }^{3}$ Max Planck Institute for Dynamics and Self-Organization, 37073 Göttingen, Germany
}

(Received 6 August 2009; accepted 14 October 2009; published online 25 November 2009)

\begin{abstract}
The flow in an enclosed rotating cylinder with a stationary lower end wall is investigated numerically. For fast rotation rates, the flow in the interior is primarily in the azimuthal direction, with an angular momentum distribution very close to that corresponding to solid-body rotation for about the inner-half radius. The differential rotation sets up a large-scale circulation that is primarily present in the boundary layers on the rotating top and sidewalls and the stationary bottom wall, with a very weak effusive component throughout the bulk interior providing a matching between the boundary layer flows on the top and bottom. The top end wall boundary layer has a profile that very closely matches the von Kármán solution for a rotating disk boundary layer; it is stable and very robust to finite disturbances for all rotation rates considered. The boundary layer on the stationary bottom end wall has a profile that agrees with the Bödewadt solution for a stationary disk with an ambient flow in solid-body rotation. This boundary layer is not robust, suffering crossflow instability to multiarmed spiral waves via a supercritical Hopf bifurcation, as well as being susceptible to axisymmetric circular waves that travel radially inward where the boundary layer profile is most inflectional. In the absence of any external forcing, the circular waves are transitory, but low amplitude forcing can sustain them indefinitely, whereas the spiral waves are essentially unaffected by the external forcing. (c) 2009 American Institute of Physics. [doi:10.1063/1.3262817]
\end{abstract}

\section{INTRODUCTION}

The boundary layer flow over a rotating disk in a quiescent fluid has frequently been used as a canonical threedimensional flow which exemplifies the crossflow instability. ${ }^{1-4}$ In this flow, the instability appears as outwardspiraling waves. These were first detected experimentally in hot-wire measurements; ${ }^{5}$ theoretical analysis together with accompanying experimental results followed later. ${ }^{6}$ The basic structure of the boundary layer on a rotating disk has much in common with that on a swept wing. The primary (tangential) component of velocity in the wing boundary layer corresponds to the azimuthal component of velocity in the disk and the secondary (crossflow) component for the wing corresponds to the radial component for the disk; see schematics of these boundary layer velocity profiles in Fig. 1 of Ref. 1 for the swept wing and Fig. 1(a) for the rotating disk. A similarity solution for the rotating disk boundary layer, one of the few exact solutions to the Navier-Stokes equations, was found by von Kármán. ${ }^{7}$

The von Kármán boundary layer is perhaps not the best analogy to the swept-wing boundary layer. The main deficiency in the analogy is that the primary flow component for the disk problem is largest at the disk, diminishes vertically through the boundary layer, and asymptotes to zero far from the disk. In contrast, for the swept wing, the primary flow component is zero at the wing and grows through the boundary layer to match the uniform profile in the free stream. However, the boundary layer on a stationary disk with the

\footnotetext{
a) Author to whom correspondence should be addressed. Electronic mail: lopez@math.asu.edu.
}

ambient flow in solid-body rotation is a much better analogy [compare the primary velocity profile for this flow in Fig. 1(b) with that of the swept wing in Fig. 1 of Ref. 1]. In all three cases, the secondary (crossflow) component vanishes at the boundary and asymptotes to zero above the boundary layer, and is inflectional in the boundary layer. Following the techniques of von Kármán, Bödewadt ${ }^{9}$ found a self-similar exact solution to the Navier-Stokes equations for this boundary layer flow. Both the von Kárman and the Bödewadt crossflows have the feature that they satisfy no slip at a wall and asymptotically tend to zero far above the wall, but there exist other crossflows that do not exhibit this feature. ${ }^{10}$

The controversy with the Bödewadt similarity solution is that it requires radial inflow from infinity to approach similarity in the boundary layer as $r \rightarrow 0 .{ }^{11}$ The speculation has been that this boundary layer flow may "break down" at some finite radius and hence Bödewadt's similarity solution may not be achieved in practice. ${ }^{12,13}$ It has been conjectured that the solution of Bödewadt would not be stable due to an inflection point instability. ${ }^{14}$

While it is nice to have exact solutions to the NavierStokes equations to analyze the stability of the flows they describe, the correspondence with physical experiments is always compromised by the finite dimensions (and hence lack of exact similarity) of the physical flows. ${ }^{15,16}$ There have been numerous studies where the similarity invariance of the basic flow in the radial direction is assumed. ${ }^{17,18}$ Nonparallel effects have been taken into account, ${ }^{19}$ but the semianalytic techniques used still suffer from the use of idealized boundary conditions, which do not match physical boundary conditions, particularly in the radial direction. In order to 
(a)

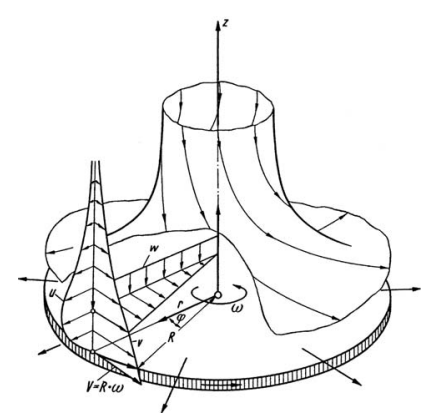

(b)

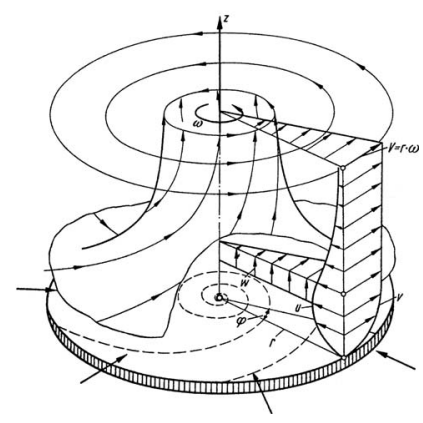

FIG. 1. Schematics of (a) von Kármán boundary layer profiles and (b) Bödewadt boundary layer profiles. From Ref. 8 [H. Schlichting and K. Gersten, Boundary-Layer Theory, 8th ed. (Springer-Verlag, Berlin, 2003)]. Copyright $(\odot 2003$ by Springer-Verlag. Reprinted with kind permission of Springer Science + Business Media.

achieve direct correspondence with any physical experiment, the full Navier-Stokes equations without imposing similarity invariance and incorporating the physical boundary conditions must be implemented. For disk boundary layer problems, this generally means that the finite disk needs to be shrouded by a cylinder.

Savaş ${ }^{20,21}$ conducted a series of experiments where initially a regime corresponding to quasisteady finite Bödewadt flow exists. The initial state consists of a filled circular cylinder in solid-body rotation of angular rotation rate $\Omega$. At time $t=0$, the cylinder is impulsively stopped. Apart from thin boundary layers on all rigid surfaces, the interior flow continues to be in solid-body rotation for some time. The stationary top and bottom end walls of the cylinder are the finite analogs of Bödewadt's disk of infinite extent. Until secondary motions grow, Savaş suggested that this flow provides a means to examine the stability of Bödewadt's solution. For a wide range of Reynolds numbers $\left(\operatorname{Re}=\Omega R^{2} / \nu\right.$, where $R$ is the cylinder radius and $\nu$ is the kinematic viscosity), he observed that in some mean sense, the profile of the boundary layer in his spindown experiments matched Bödewadt's profiles (at least for about one rotation period). He also observed that for $\operatorname{Re} \approx 10^{4}$, the boundary layer supported a series of circular (i.e., axisymmetric) waves which first become apparent at a radius $r \approx R / 2$ and propagate radially inward, but do not reach the axis. In the vicinity of the axis, the boundary layer is of Bödewadt type (i.e., the velocity profiles in the experimental flow match the similarity profiles).

Lopez and Weidman ${ }^{22}$ repeated the experiments of Savaş, ${ }^{21}$ reproducing the radially inward traveling circular waves in the disk boundary layer, and also simulated these states using the Navier-Stokes equations. They further showed numerically that if instead of impulsively stopping the entire cylinder, only the end walls are stopped while the cylindrical sheath continues rotating, then the development of the disk boundary layer and its inflectional instability leading to the formation of the circular waves is essentially the same, but without the large disturbances from the sidewall due to the whole flow spinning down. In a follow-up study, ${ }^{23}$ the finite Bödewadt flow where only a single disk is

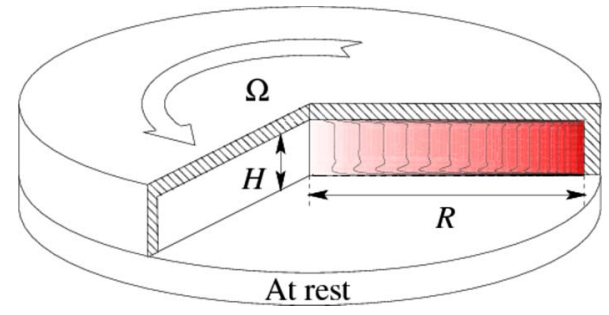

FIG. 2. (Color online) Schematic of the finite Bödewadt flow. The inset shows the vortex lines (contours of $r v$ ) of the flow for $\operatorname{Re}=5 \times 10^{4}$ and $\gamma$ $=0.2$, at steady state.

stopped and the rest of the cylinder continues to rotate was investigated both numerically and experimentally, capturing the radially traveling axisymmetric waves, but only as transients. The numerics in that study were restricted to solving the axisymmetric Navier-Stokes equations, and so could not address the spiral waves.

In this paper we present direct numerical simulations of the flow in an enclosed rotating cylinder with a stationary lower disk. This configuration provides the closest analogy of the Bödewadt problem in a finite geometry. We find that multiarmed spiral waves emerge following a global instability of the basic state via a supercritical Hopf bifurcation, whereas the aforementioned axisymmetric waves are always transient, corresponding to a convective instability localized in the stationary end wall boundary layer where it is most inflectional. When low amplitude forcing is continuously applied by modulating the rotation rate of the top and sidewalls, the system behaves like a spatial amplifier and supports self-sustained axisymmetric waves. These are present even beyond the onset of the global instability, where they coexist with the multiarmed spirals.

\section{GOVERNING EQUATIONS AND NUMERICAL METHODS}

Consider the flow in a circular cylinder of height-toradius aspect ratio $\gamma=H / R$ completely filled with a fluid of kinematic viscosity $\nu$. The cylinder and the top end wall rotate with angular speed $\Omega$ while the bottom end wall is stationary. A schematic of the flow system is shown in Fig. 2. The Navier-Stokes equations, nondimensionalized using the radius $R$ as the length scale and $1 / \Omega$ as the time scale, are

$$
\left(\partial_{t}+\mathbf{u} \cdot \nabla\right) \mathbf{u}=-\nabla p+\frac{1}{\operatorname{Re}} \nabla^{2} \mathbf{u}, \quad \nabla \cdot \mathbf{u}=0,
$$

where $\mathbf{u}=(u, v, w)$ is the velocity field in polar coordinates $(r, \theta, z) \in[0,1] \times[0,2 \pi] \times[0, \gamma]$ and $p$ is the kinematic pressure. There are only two governing parameters, the aspect ratio $\gamma$ and the Reynolds number $\operatorname{Re}=\Omega R^{2} / \nu$. The boundary conditions are no slip: on the rotating cylinder sidewall $(u, v, w)=(0,1,0)$, on the rotating top end wall $(u, v, w)=(0, r, 0)$, and on the bottom stationary end wall $(u, v, w)=(0,0,0)$. The idealized boundary conditions are discontinuous at the junctions where the rotating cylinder meets the stationary bottom end wall at $(r=1, z=0)$. In a physical experiment there is a small but finite gap at this junction where the azimuthal velocity adjusts rapidly to zero. 
For an accurate use of spectral techniques, a regularization of this discontinuity is implemented of the form

$$
v(r, \theta, 0, t)=\exp \left(\frac{r-1}{\epsilon}\right),
$$

where $\epsilon$ is a small parameter that mimics the small physical gaps (we have used $\epsilon=0.003$ ). The use of $\epsilon \neq 0$ regularizes the otherwise discontinuous boundary condition; see Ref. 24 for further details on the use of this technique in spectral codes.

The governing equation (1) has been solved using a second-order time-splitting method. ${ }^{25}$ The spatial discretization is via a Galerkin-Fourier expansion in $\theta$ and a Chebyshev collocation in $r$ and $z$. The spectral solver used here is based on a previous scheme recently tested and used in a wide variety of flows in enclosed cylinders. ${ }^{26-28}$ For the solutions presented here, we have used up to $n_{r}=128$ and $n_{z}$ $=64$ Chebyshev modes in the radial and axial directions and up to $n_{\theta}=360$ azimuthal Fourier modes, depending on $\gamma, \operatorname{Re}$, and the azimuthal wavenumber of the computed solutions, ensuring that the trailing coefficients of the spectral expansion are at least five orders of magnitude smaller than the leading coefficients. Time steps of the order $\delta t=10^{-3}$ have been required for numerical stability and accuracy of the second-order temporal scheme.

\section{BASIC STATE}

The basic state has been extensively described and discussed previously, ${ }^{23,22,29}$ and only a brief overview is presented here. For any aspect ratio $\gamma$, if Re is large enough the boundary layers on the disks are separated by an interior region which is nominally rotating with a near solid-body rotation distribution of angular momentum, except close to the cylinder sidewall. However, it is never exactly solid-body rotation as the flow on the top disk boundary layer is centrifuged radially outward while that on the bottom disk boundary layer is drawn radially inward. Over a considerable radial extent (about $80 \%$ ), the flow in the interior provides a match between the two boundary layer flows, ${ }^{30}$ the radial velocity in the interior is very small and the angular momentum distribution is almost $z$-independent, but does not correspond to solid-body rotation. Figure 3 shows the streamlines $\psi$, the vortex lines $r v$, and the azimuthal component of vorticity $\eta$ for the steady axisymmetric basic state at $\operatorname{Re}=5 \times 10^{4}$ and $\gamma=0.2$. For axisymmetric flow, these quantities are related to the velocity by $\mathbf{u}=(u, v, w)=(-\partial \psi / \partial z, r v, \partial \psi / \partial r) / r$ and $\nabla$ $\times \mathbf{u}=(-\partial r v / \partial z, r \eta, \partial r v / \partial r) / r$. As can be seen from the streamlines, the sidewall boundary layer provides the conduit closing the circulation of flow traveling radially inward in the bottom stationary disk boundary layer, effusing vertically up out of that layer and into the top rotating disk layer which is then centrifuged radially outward and returned to the bottom layer along the sidewall layer. Note that the vortex lines are drawn using a quadratic distribution of contour levels for $r v$. If the flow was in solid-body rotation, then $r v \propto r^{2}$ and the vortex lines drawn in this way would be uniformly spaced in $r$; they are for $r<0.5$, but for $r>0.5$ there is a considerable departure from solid-body rotation. (a) streamlines $\psi$

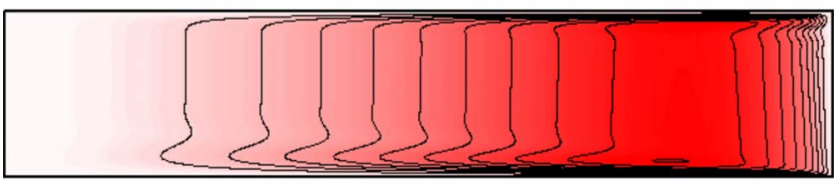

(b) vortex lines $r v$

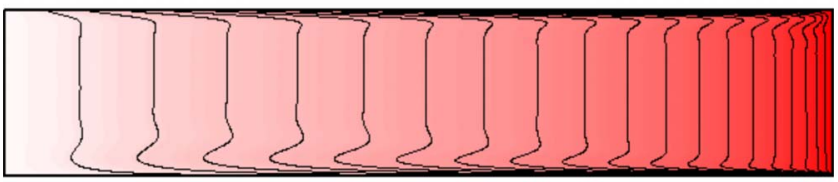

(c) azimuthal vorticity $\eta$

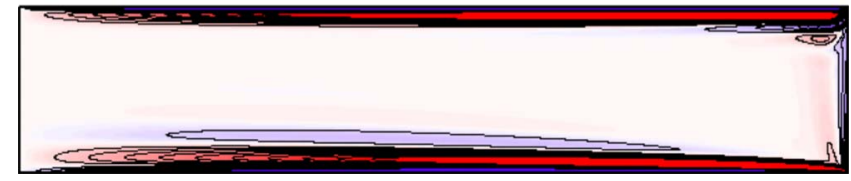

FIG. 3. (Color online) Contours of streamlines, vortex lines, and azimuthal vorticity for the steady basic state at $\operatorname{Re}=5 \times 10^{4}$ and $\gamma=0.2$ using $n_{r} \times n_{z}$ $=96 \times 48$ and $\delta t=5 \times 10^{-3}$; the contours are in the ranges $\psi \in[0,0.004]$, $r v \in[0,1]$, and $\eta \in[-5,5]$; the $\psi$ and $\eta$ contours are uniformly spaced and the $r v$ contours are quadratically spaced.

The vortex line plot [contours of $r v$, shown in Fig. 3(b)] clearly shows the typical characteristics of rotating disk boundary layers on the top and stationary disk boundary layers on the bottom. Note however that while the boundary layer thickness along the top rotating disk is quite uniform, the boundary layer thickness on the bottom stationary disk varies considerably with $r$, thickening as $r$ is reduced [this is even more clearly evident in the contour plot of the azimuthal vorticity $\eta$, shown in Fig. 3(c)], while at a fixed $r$ location it has the characteristic oscillatory structure of the Bödewadt solution. As has been previously noted, ${ }^{23,29}$ this layer has the expected square-root scaling, as shown in Fig. 4. This figure also serves to illustrate the degree to which the layer is resolved using $n_{r} \times n_{z}=96 \times 48$ Chebyshev modes; there are about four collocation points in the viscous sub-

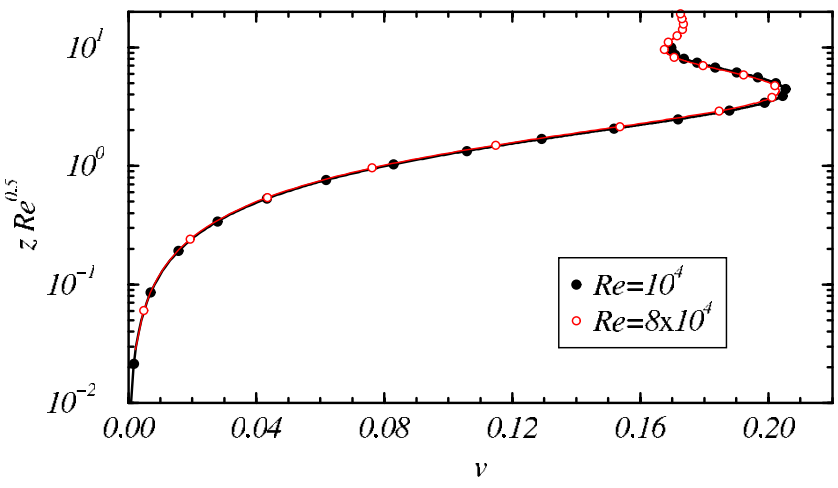

FIG. 4. (Color online) Profiles of the azimuthal velocity $v$ in the Bödewadt boundary layer at $r=0.5$ for $\gamma=0.2$ and $\mathrm{Re}=1 \times 10^{4}$ and $8 \times 10^{4}$ (the $\mathrm{Re}$ $=8 \times 10^{4}$ is computed in the axisymmetric subspace), with $n_{r} \times n_{z}=96 \times 48$. The vertical direction $z$ has been scaled with $\mathrm{Re}^{0.5}$, collapsing the profiles. The Chebyshev collocation points are indicated by the filled $\left(\operatorname{Re}=10^{4}\right)$ and open $\left(\operatorname{Re}=8 \times 10^{4}\right)$ symbols. 
layer and about another six in the log layer for the range of $\operatorname{Re} \in\left[10^{4}, 8 \times 10^{4}\right]$ that is principally considered in this paper. The thickening of the Bödewadt layer with decreasing $r$ and the scaling of the thickness with $\mathrm{Re}^{-0.5}$ has also been observed in experiments at $\gamma=0.05,{ }^{31}$ emphasizing the nonparallel nature of the boundary layer on the stationary disk.

\section{LINEAR STABILITY OF THE BASIC STATE: SPIRAL WAVES}

The stability of finite von Kármán and Bödewadt flows has often been investigated by making a parallel-flow approximation, i.e., taking the vertical velocity profile at a fixed radius and using it to define a self-similar flow of infinite radial extent. This is then repeated at different radial stations to determine the global stability of the flow, and one can infer that absolute instability in a finite region is necessary for global instability. However, the departure from solidbody rotation for the interior of the finite domain results in boundary layers on the disks that, while they locally have the expected vertical profiles, do not have self-similar radial distributions and are not parallel. Thus, while a local analysis does give insight into the stability of the boundary layer, it has obvious limitations due the finite nature of the actual flow of interest, and has primarily been used to avoid the computational expense of the analysis with physical boundary conditions. ${ }^{32}$

Here, the global linear stability analysis is performed via time evolution of the Navier-Stokes equations. First, a steady axisymmetric basic state is computed at some point in parameter space. Its stability is determined by introducing small random perturbations into all azimuthal Fourier modes. For sufficiently small perturbations, the nonlinear couplings between Fourier modes are negligible (below round-off numerical noise) and the growth rates (real parts of the eigenvalues) and structure of the eigenfunctions corresponding to the fastest growing perturbation at each Fourier mode emerge from time evolution. This is tantamount to a matrixfree generalized power method in which the actions of the Jacobian matrices for the perturbations are given by time integration of the Navier-Stokes equations with the aforementioned initial conditions. This direct numerical technique is very efficient as the exponential growth or decay of the perturbations is established in a relatively short evolution time (there is no need to evolve the disturbances until they saturate nonlinearly).

The stability of the basic states to three-dimensional perturbations at a few values of Re is determined by monitoring the growth rates of the kinetic energy of each azimuthal Fourier mode. For each Fourier mode, we find a pair of Re values at which the growth rate changes sign and then we refine in Re (computing new basic states at each intermediate Re value). With about four or five growth rates bracketing zero growth rate, linear interpolation is used to estimate the critical Re for the given Fourier mode. For $\gamma=0.2$, the critical Re for the most dangerous Fourier modes are plotted in Fig. 5. We find that the basic state first loses stability at $\mathrm{Re}_{\mathrm{c}}=51735$ (based on the $128 \times 64$ resolution; the value for the lower $96 \times 48$ resolution is 51743 ) to an $m=32$ spiral

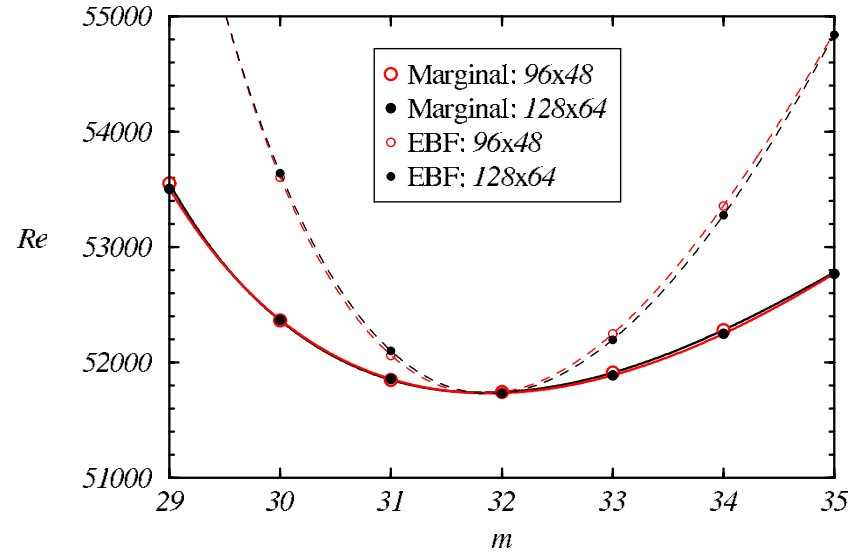

FIG. 5. (Color online) Marginal stability curves (solid lines) for $\gamma=0.2$ with two resolutions, $n_{r} \times n_{z}=96 \times 48$ and $128 \times 64$, showing the critical Re at which the axisymmetric basic state loses stability via supercritical Hopf bifurcations to spiral rotating waves with azimuthal wavenumber $m$. Also shown are the corresponding EBF stability curves (dashed lines), estimated from the marginal stability curves using the classical prediction $\operatorname{Re}_{\mathrm{EBF}}(m)$ $=3\left[\operatorname{Re}_{\mathrm{M}}(m)-\mathrm{Re}_{\mathrm{c}}\right]+\mathrm{Re}_{\mathrm{c}}$. The lines are a guide for the eye as the wavenumbers $m$ are integers.

rotating wave. For $\operatorname{Re}>\operatorname{Re}_{c}$, the basic state is unstable to spiral modes within a band of wavenumbers, and a subset of these within an Eckhaus-Benjamin-Feir (EBF) band will be stable. In Fig. 5 we have included an estimate of the EBF stability boundary based on the classic prediction ${ }^{33}$ $\operatorname{Re}_{\mathrm{EBF}}(m)=3\left[\operatorname{Re}_{\mathrm{M}}(m)-\mathrm{Re}_{\mathrm{c}}\right]+\operatorname{Re}_{\mathrm{c}}$, where $\operatorname{Re}_{\mathrm{M}}(m)$ is the critical $\mathrm{Re}$ for a given wavenumber $m$ and $\mathrm{Re}_{\mathrm{c}}$ is the minimum critical Re over all $m$. EBF stability boundaries have been measured experimentally for a related flow between a stationary and a rotating disk but with a stationary shroud, ${ }^{34}$ as well as in experiments ${ }^{35}$ and numerical simulations ${ }^{27}$ of rotating convection.

We have evolved the $m=32$ spiral wave to full nonlinear saturation at $\operatorname{Re}=6 \times 10^{4}$; this takes several dozen cylinder rotation times using the axisymmetric basic state at this $\mathrm{Re}$ plus a small multiple (of magnitude $\sim 10^{-6}$ ) of the $m=32$ Fourier component from the direct stability analysis (which provides a very good approximation to the corresponding Hopf eigenmode). Figure 6(a) shows an instantaneous contour plot of the vertical velocity in an $(r, z)$-meridional section; the only departure from the basic state is the presence of what appear to be a series of counter-rotating cells in the stationary disk boundary layer for $r \in(0.5,0.8)$. A horizontal cross section through the layer at $z=0.05 \gamma$ shows that these cells actually correspond to cuts through several arms of an $m=32$ spiral wave. These emerge at a supercritical symmetry-breaking Hopf bifurcation resulting from the strong inflectional nature of the velocity profile at about $75 \%$ radius near the stationary disk. The spiral wave is a rotating wave; it is a three-dimensional time-periodic state whose spatial structure is time invariant as it precesses uniformly. In this case, the precession is prograde with the top and shroud rotation, with a precession frequency $\omega_{p} \approx 0.156$. This can be easily measured from the space-time plot in Fig. 6(c), showing $w(t, r)$ at $z=0.05 \gamma$ and $\theta=0$ for five disk rotations $(t$ $=10 \pi)$ and the whole radius. 
(a)

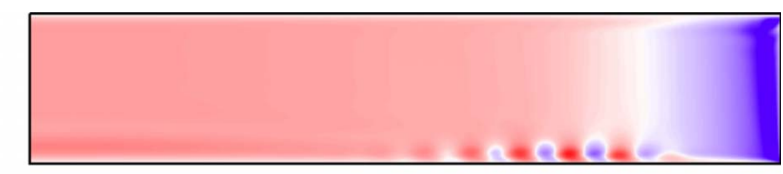

(b)

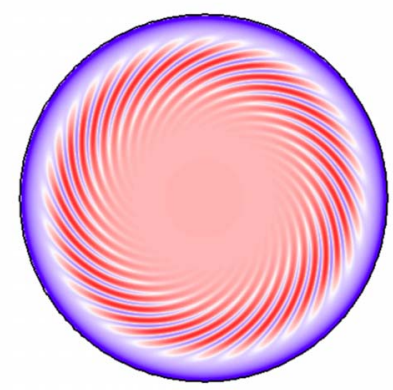

$(c)$

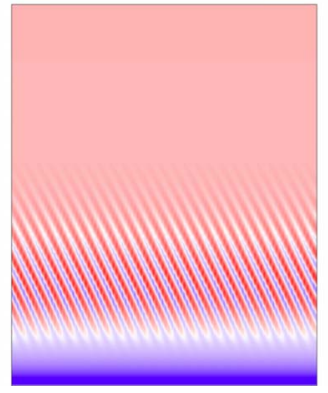

FIG. 6. (Color online) Snapshots of the vertical velocity $w$ of a $m=32$ spiral wave at $\operatorname{Re}=6 \times 10^{4}, \gamma=0.2$ in (a) a meridional section $r \times z \in[0,1]$ $\times[0,0.2]$, and (b) a horizontal section at $z=0.05 \gamma$, together with (c) a spacetime plot of $w$ at $z=0.05 \gamma$ with $r \in[0,1]$ (vertical axis, with $r$ increasing from top to bottom) and $t \in[0,10 \pi]$ (horizontal axis, with $t$ increasing from left to right); red (dark) is positive and blue (light) is negative, with pure white being zero (enhanced online). [URL: http://dx.doi.org/10.1063/ 1.3262817.1] [URL: http://dx.doi.org/10.1063/1.3262817.2]

\section{AXISYMMETRIC CIRCULAR WAVES}

The global stability results of Sec. IV show that the steady axisymmetric basic state becomes unstable to spiral waves via a Hopf bifurcation at about $\operatorname{Re}=5.17 \times 10^{4}$. Apparently, this contradicts most of the previous investigations which report that axisymmetric circular traveling waves are the primary mode of instability for substantially lower Re. In order to shed light on the origin of these circular waves, we have performed highly resolved computations restricted to the axisymmetric subspace so that interaction with the threedimensional spiral waves is avoided. Our results indicate that the steady basic state is linearly stable with respect to axisymmetric perturbations, at least up to the highest Re considered, $\operatorname{Re}=10^{5}$. However, for $\operatorname{Re}>10^{3}$ transient circular waves exist in the stationary disk boundary layer. These have been previously reported and analyzed in detail, ${ }^{23}$ and have always been found to be transient, with their lifetime increasing with Re.

The circular waves propagate radially inward in the Bödewadt layer, intensify at about midradius, and then decay as they approach the axis. As a result of the decay, there is no apparent feedback on the basic state even though there is a large-scale meridional circulation, and the behavior in the Bödewadt layer is a characteristic of an unstable, nonparallel open flow. In related experiments with $\gamma \approx 0.05$ and $\mathrm{Re}$ $\in\left[1.6 \times 10^{4}, 8 \times 10^{4}\right]$, this type of behavior of the circular waves has also been reported. ${ }^{31}$ Those same experiments ${ }^{31}$ also report that the onset of the circular waves is sensitive to extraneous noise, and noted that changing the motor used to drive the rotation of the system resulted in about a 50\% increase in the lowest $\mathrm{Re}$ at which the circular waves were observed, and yet that the onset of spiral waves (which had about 31 arms) did not appear to be sensitive to the noise level.
We note that in our present simulations using a highly resolved spectral technique, as well as in the earlier simulations using finite differences, ${ }^{23}$ evolutions starting either from rest or from a solution at one Re conducted at a different Re always lead to undulations in the Bödewadt layer, and if $\operatorname{Re}$ is sufficiently large ( $\operatorname{Re} \geq 10^{4}$, depending on $\gamma$ ), these undulations form circular waves. In all cases (we have explored Re up to $10^{5}$ ), these waves eventually die out.

\section{RESPONSE TO FINITE IMPULSIVE CHANGE IN ROTATION RATE}

The response to impulsive changes in the rotation rate has been seen to always involve the generation of a train of circular waves which typically die out after a few rotations of the system. Such responses were reported from axisymmetric simulations ${ }^{23}$ for $\operatorname{Re}=10^{4}$ and $\gamma=1$, with impulsive changes in Re up to $\pm 50 \%$, and in experiments ${ }^{31}$ with $\gamma \approx 0.048$, transient circular waves were observed after impulsive perturbations (magnitudes of the perturbations were not reported) for $\operatorname{Re}>3 \times 10^{4}$, and those waves persisted whether or not impulsive perturbations were introduced into the system for $\operatorname{Re}>4.8 \times 10^{4}$. Reference 31 reports that their experiments are unable to conclusively say whether the circular waves are due to a transition from locally convective to locally absolute instability in the boundary layer or due to noise-sustained structures in a still convectively unstable regime. Of course, in any experiment, there is an ever present noise which typically increases with $\mathrm{Re}$, and so wellresolved accurate simulations are necessary in making this distinction.

We reiterate that for all Re considered, in the absence of external forcing, the circular waves are transitory. Now, we consider impulsively changing Re by $5 \%$ for two cases, one for $\mathrm{Re}=5 \times 10^{4}$, which is just below the onset of the spiral waves where the steady axisymmetric basic state (shown in Fig. 3) is stable, and another at $\operatorname{Re}=6 \times 10^{4}$, where the flow eventually settles onto the spiral wave state shown in Fig. 6. Figure 7 (together with the online movie) shows the response to the $5 \%$ impulsive change in $\operatorname{Re}$ at $\operatorname{Re}=5 \times 10^{4}$; the initial condition is the basic state, and within about the first rotation time, a circular wave train appears. Within about ten rotation times, these have completely died out and we are left with the axisymmetric basic state at the new value $R e=5.25$ $\times 10^{4}$. At this Re, the basic state is linearly unstable to spiral waves, but these grow very slowly, requiring several dozen of rotation times to appear. Furthermore, the impulsive change in $\mathrm{Re}$ is an axisymmetric forcing and so does not directly affect the spiral modes (other than to change the basic state to that of a higher Re at which the spiral waves have positive linear growth rate). On subjecting a spiral state to an impulsive $5 \%$ change in Re, we observe a very similar response with the spiral wave being largely unaffected by the transient circular wave train, as seen in Fig. 8 and the accompanying online movie.

The susceptibility of the Bödewadt layer to the transient circular waves suggests that small extraneous perturbations, which are ubiquitous in any physical experiment, can be expected to lead to similar noise-sustained boundary layer 


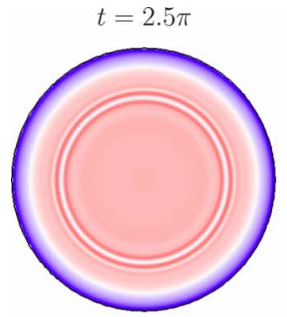

$7.5 \pi$

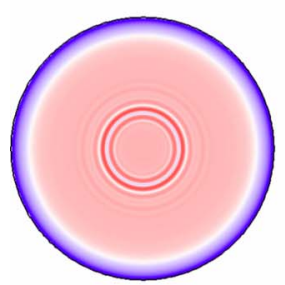

$20.0 \pi$
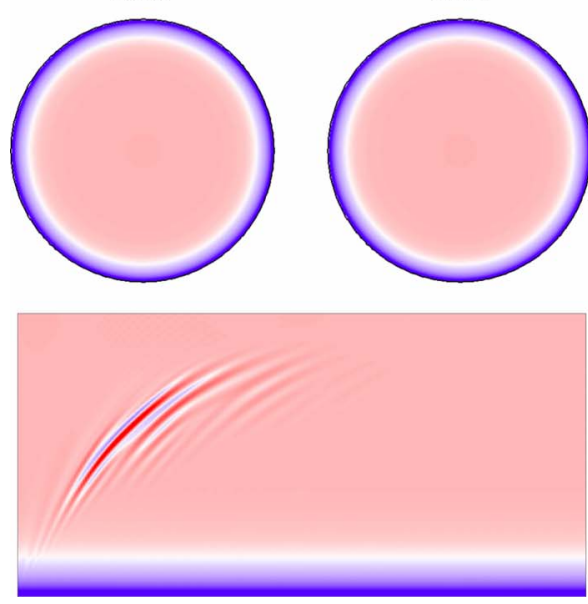

FIG. 7. (Color online) Snapshots of the vertical velocity at $z=0.05 \gamma$ at times as indicated following an impulsive change in Re from $5.00 \times 10^{4}$ to 5.25 $\times 10^{4}$ at $t=0$. Also shown is a space-time diagram of the vertical velocity at $z=0.05 \gamma$ with $r \in[0,1]$ (vertical axis, with $r$ increasing from top to bottom) and $t \in[0,25 \pi]$ (horizontal axis, $t$ increasing from left to right) (enhanced online). [URL: http://dx.doi.org/10.1063/1.3262817.3] [URL: http://dx.doi.org/10.1063/1.3262817.4]

eruptions, even in parameter regimes where in the absence of noise the smooth basic state would be stable. It is then necessary to assess the effect of sustained perturbations instead of impulsive changes that are almost instantaneous. As any perturbation can be expanded in temporal Fourier components, a natural and simple candidate for sustained perturbations is a harmonic modulation of the rotation rate; this is analyzed in Sec. VII.

\section{RESPONSE TO HARMONIC MODULATION}

In this section we consider a harmonic modulation of the rotation rate of the form

$$
\Omega(t)=\Omega_{0}\left[1+A \sin \left(\omega_{f} t\right)\right],
$$

where the nondimensional parameters $A$ and $\omega_{f}$ are the amplitude and frequency of the forcing. The mean rotation rate $\Omega_{0}$ is used to set the time scale $1 / \Omega_{0}$ and the mean Reynolds $\operatorname{Re}=\Omega_{0} R^{2} / \nu$. The problem now has four independent nondimensional parameters $(\operatorname{Re}, \gamma, A$, and $\omega)$ and a thorough ex-

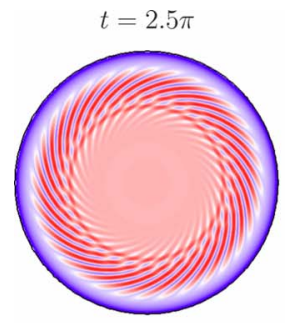

$7.5 \pi$

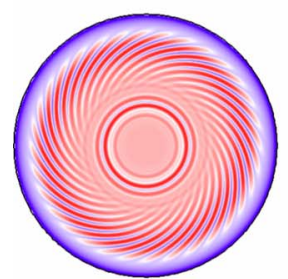

$20.0 \pi$
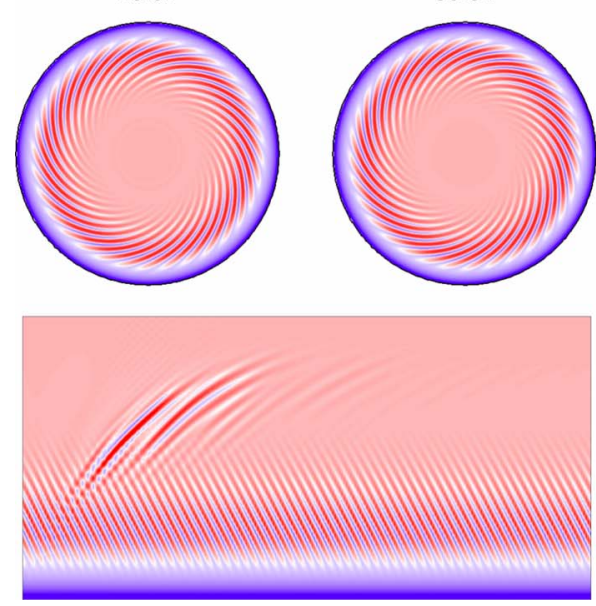

FIG. 8. (Color online) Snapshots of the vertical velocity at $z=0.05 \gamma$ at times as indicated following an impulsive change in Re from $6.0 \times 10^{4}$ to 6.3 $\times 10^{4}$ at $t=0$. Also shown is a space-time diagram of the vertical velocity at $z=0.05 \gamma$ with $r \in[0,1]$ (vertical axis, with $r$ increasing from top to bottom) and $t \in[0,25 \pi]$ (horizontal axis, $t$ increasing from left to right) (enhanced online). [URL: http://dx.doi.org/10.1063/1.3262817.5] [URL: http://dx.doi.org/10.1063/1.3262817.6]

ploration of parameter space is extremely costly. As we are interested in small perturbations of the rotation rate, we have fixed $A=0.05$, a $5 \%$ modulation amplitude. We have also set the forcing frequency to one, $\omega_{f}=1$, for simplicity; as the state without forcing is steady, we do not expect any resonance effect between $\omega_{f}$ and $\Omega_{0}$.

Figure 9 shows the resulting solution at a mean $\mathrm{Re}=5$ $\times 10^{4}$ and $\gamma=0.2$, where the unforced base state is stable. We observe that the solution exhibits a persistent train of circular waves, synchronous with the forcing. These circular waves have characteristics very similar to the waves resulting from impulsive changes in $\mathrm{Re}$, but now they are permanent due to the continual harmonic acceleration and deceleration of the upper disk and shroud rotation. The waves appear at midradius and propagate radially inward within the stationary bottom disk boundary layer, and disappear before reaching the cylinder axis.

One may wonder if these states with permanent circular waves result from a bifurcation of a synchronous basic state without waves, or if they are an inherent feature of the basic 
(a)

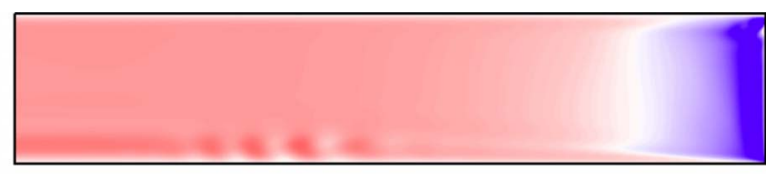

(b)

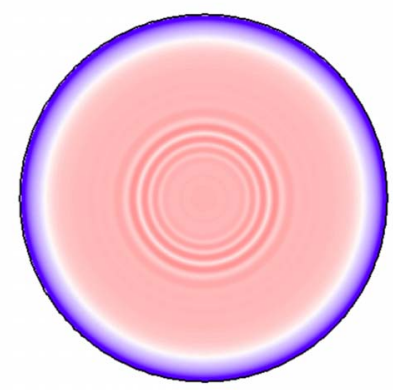

$(c)$

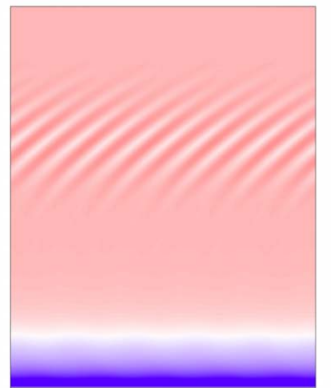

FIG. 9. (Color online) Snapshots of the vertical velocity $w$ of a synchronous axisymmetric state in (a) a meridional section and (b) a horizontal section at $z=0.05 \gamma$ at a mean $\operatorname{Re}=5 \times 10^{4}$ modulated by $5 \%$ at frequency $\omega_{f}=1.0$, together with (c) a space-time plot of $w$ at $z=0.05 \gamma$ with $r \in[0,1]$ (vertical axis, with $r$ increasing from top to bottom) and $t \in[0,10 \pi]$ (horizontal axis, with $t$ increasing from left to right) (enhanced online). [URL: http://dx.doi.org/10.1063/1.3262817.7] [URL: http://dx.doi.org/10.1063/1.3262817.8]

state in the forced case, regardless of the amplitude of the forcing. In order to address this issue, we have computed solutions at $\operatorname{Re}=5 \times 10^{4}, \gamma=0.2$, and $\omega_{f}=1$ for a range of forcing amplitude $A$ covering several orders of magnitude. We have found evidence of the circular wave train for all $A$, and as expected the amplitudes of the waves relative to the mean flow diminishes with $A$. We have not found any evidence of a change in flow mode with decreasing $A$, thus we conclude that the train of synchronous circular waves emerges smoothly from the unforced $A=0$ state.

Figure 10 shows the solution of the periodically forced

(a)

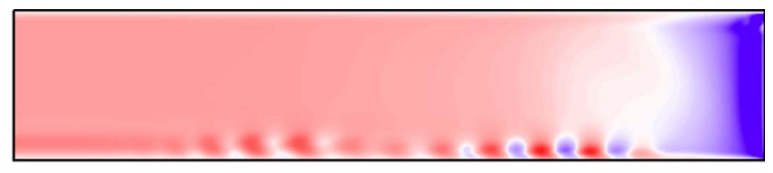

(b)

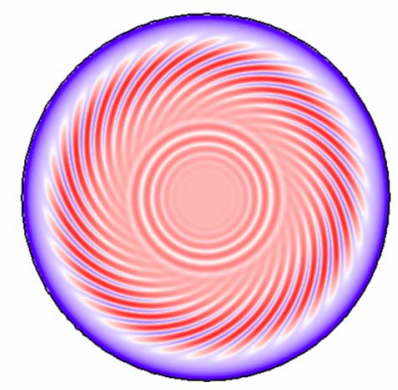

$(c)$

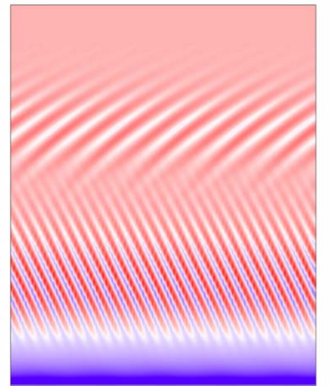

FIG. 10. (Color online) Snapshots of the vertical velocity $w$ of a synchronous axisymmetric state in (a) a meridional section and (b) a horizontal section at $z=0.05 \gamma$ at a mean $\operatorname{Re}=6 \times 10^{4}$ modulated by $5 \%$ at frequency $\omega_{f}=1.0$, together with (c) a space-time plot of $w$ at $z=0.05 \gamma$ with $r$ $\in[0,1]$ (vertical axis, with $r$ increasing from top to bottom) and $t$ $\in[0,10 \pi]$ (horizontal axis, with $t$ increasing from left to right) (enhanced online). [URL: http://dx.doi.org/10.1063/1.3262817.9] [URL: http://dx.doi.org/10.1063/1.3262817.10] case (at $\gamma=0.2, A=0.05$, and $\omega_{f}=1$ ) for a higher mean Reynolds number $\operatorname{Re}=6 \times 10^{4}$, where the spiral waves have already bifurcated from the base state in the unforced case. We observe that the spiral waves and the circular waves coexist with very small mutual interference. The pattern is almost identical to the linear superposition of the two previously obtained solutions, the pure spiral wave in the unforced case (Fig. 6) and the permanent circular waves in the forced case (Fig. 9). In fact, in the periodically forced case the spiral waves bifurcate from the base state with circular waves at almost the same Re value as in the unforced case. This is a Neimark-Sacker bifurcation of the periodic base state, resulting in a quasiperiodic solution with two frequencies: the forcing frequency associated with the synchronous circular waves and the precession frequency of the spiral waves. As the periodic base state has $S O(2)$ symmetry, the Hopf frequency associated with the Neimark-Sacker bifurcation corresponds to a precession frequency, and the $S O(2)$ symmetry inhibits resonant behavior between the two frequencies. ${ }^{36}$

\section{DISCUSSION AND CONCLUSIONS}

There has been much recent interest in transition processes in rotor-stator configurations. This related problem consists of a rotating disk and a stationary disk, shrouded by a cylindrical sidewall, and there may also be an inner cylindrical hub. ${ }^{37-42}$ However, the motivation for these studies comes from applications in turbomachinery rather than interests in crossflow boundary layer instabilities, and the setups studied typically have the gap between the two disks being a very small fraction of their radius and the cylindrical shroud is stationary. These characteristics of the rotor-stator setup lead to the boundary layer on the rotating disk being turned into the interior by the presence of the stationary shroud, forming a free shear layer that under some conditions becomes unstable to azimuthal waves ${ }^{43}$ or the free shear layer may be drawn (via Ekman suction type processes) into either the boundary layer on the rotating or stationary disk or even periodically flipping between the two. ${ }^{29}$ Recent results ${ }^{44}$ in a rotor-stator annular geometry with rotating inner hub and stationary outer shroud and a sufficient gap between the disks so that their boundary layers are well separated suggest that the stationary outer shroud introduces a permanent perturbation, which destabilizes the Bödewadt-like layer on the stator and induces spiral patterns. In that flow, both the axisymmetric and the spiral waves are much larger than the boundary layer thickness on either disk, and are not seen to originate as disk boundary layer instabilities. ${ }^{44}$ The point is that the rotor-stator configuration with the stationary shroud leads to transition processes that are not directly related to the crossflow instability; that requires the shroud to be rotating so that the interior flow is close to solid-body rotation and the stationary disk boundary layer is of Bödewadt type.

A series of experiments in a shallower system with a rotating shroud $(\gamma=0.05$, compared to our $\gamma=0.2)$ (Ref. 31) investigated the nature of the circular waves by subjecting the system to harmonic modulations of the rotation rate, impulsive changes in the rotation rate, as well as the ambient experimental noise without any other imposed forcing, in an 
attempt to address whether the circular waves, which above a certain Re persisted indefinitely in the experiments, are noise sustained or self-sustained. They conjectured that at small Re they are noise sustained but become self-sustained at large enough Re. However, they did comment that further experiments are needed to test their conjecture. Our numerical results indicate that the circular waves are indeed noise sustained; i.e., unforced the circular waves always eventually decay. However, below the onset of the spiral instability, the flow exhibits large transient growth in response to impulsive perturbations, such as suddenly changing the Reynolds number. When perturbations are applied continuously in time, the system behaves as a spatial amplifier ${ }^{45-47}$ and when this forcing is harmonic a synchronous train of circular waves persists. The behavior of spatial amplifiers has been classically explained in terms of local convective instability ${ }^{45}$ and has also been interpreted as arising from the non-normality of global modes in nonparallel flows. ${ }^{48}$ Here we find that the dominant effect leading to persistent circular waves is due to a response to the continuously changing basic flow due to the imposed forcing.

We have only been able to obtain solutions with persistent trains of circular waves when the rotation of the top disk and shroud is modulated. Furthermore, the onset of the circular waves in the modulated case does not appear to be due to a bifurcation from a state without circular waves, but rather a smooth onset with increasing amplitude of modulation $A$ for fixed Re or increasing Re with fixed $A$. This observation is consistent with other pattern forming problems, such as Taylor-Couette and vortex breakdown flows, where the idealized problems are cast as having a direction of infinite extent along which the base flow is assumed invariant. In such synthetic problems, a bifurcation occurs that leads to either periodic cellular patterns (such as Taylor vortices when there is no mean flow in the invariant direction) or localized structures (such as recirculations zones in the vortex breakdown problem which does have mean flow in the invariant direction). In the Bödewadt problem, the synthetic idealization treats the radial direction $r$ as an infinite selfsimilar direction, and then circular waves are found to bifurcate when a local analysis in $r$ is made. However these synthetic problems are never realized physically, and when realistic geometries and boundary conditions are implemented, one finds a smooth transition from the featureless flow at vanishingly small $\mathrm{Re}$ to structured flow as $\mathrm{Re}$ is increased, without any bifurcation being responsible for the appearance of flow structure.

In conclusion, the flow analyzed exhibits two very different kinds of waves: the circular waves, to which the Bödewadt boundary layer is very sensitive, but these waves always decay in the absence of external forcing and are therefore noise sustained; and the spiral waves that correspond to a supercritical Hopf bifurcation breaking the $S O(2)$ symmetry (axisymmetry of the basic state), and are rather insensitive to external forcings. Both waves manifest themselves inside the Bödewadt boundary layer near where the mean boundary layer profile is most inflectional. When they coexist, there is very little interaction between the two classes of waves, with the circular waves propagating radi- ally inward from the region of maximal inflection and the spiral arms extending radially outward from about the same point.

\section{ACKNOWLEDGMENTS}

This work was supported in part by the U.S. National Science Foundation under Grant No. DMS-0505489, the Spanish Ministry of Education and Science under Grant Nos. FIS2007-61585 and HA2005-0087, the Catalonian Government under Grant No. SGR-00024, and the Korean Science and Engineering Foundation WCU under Grant No. R322009-000-20021-0. M.A. was funded by the Max Planck Society. Computational resources of the ASU Fulton High Performance Computing Initiative are greatly appreciated.

${ }^{1}$ W. Saric, H. Reed, and E. White, "Stability and transition of threedimensional boundary layers," Annu. Rev. Fluid Mech. 35, 413 (2003).

${ }^{2}$ P. J. Zandbergen and D. Dijkstra, "Von Kármán swirling flows," Annu. Rev. Fluid Mech. 19, 465 (1987).

${ }^{3}$ H. Reed and W. Saric, "Stability of three-dimensional boundary layers," Annu. Rev. Fluid Mech. 21, 235 (1989).

${ }^{4}$ H. Othman and T. C. Corke, "Experimental investigation of absolute instability of a rotating-disk boundary layer," J. Fluid Mech. 565, 63 (2006).

${ }^{5} \mathrm{~N}$. Smith, "Exploratory investigation of laminar boundary layer oscillations on a rotating disk," NACA Technical Report No. 1227, 1946 (available online at http://naca.central.cranfield.ac.uk/reports/1947/naca-tn1227.pdf).

${ }^{6}$ N. Gregory, J. T. Stuart, and W. S. Walker, "On the stability of three dimensional boundary layers with application to the flow due to a rotating disk," Philos. Trans. R. Soc. London, Ser. A 248, 155 (1955).

${ }^{7}$ T. von Kármán, "Über laminare und turbulente Reibung," Z. Angew. Math. Mech. 1, 2605 (1921).

${ }^{8} \mathrm{H}$. Schlichting and K. Gersten, Boundary-Layer Theory, 8th ed. (SpringerVerlag, Berlin, 2003).

${ }^{9}$ U. T. Bödewadt, "Rotary currents on fixed grounds," Z. Angew. Math. Mech. 20, 241 (1940).

${ }^{10} \mathrm{P}$. D. Weidman, "New solutions for laminar boundary layers with cross flow," ZAMP 48, 341 (1997).

${ }^{11}$ H. P. Greenspan, The Theory of Rotating Fluids (Cambridge University Press, London, 1968).

${ }^{12}$ F. K. Moore, "Three-dimensional boundary layer theory," Adv. Appl. Mech. 4, 159 (1956).

${ }^{13}$ M. H. Rogers and G. N. Lance, "The boundary layer on a disc of finite radius in a rotating fluid," Q. J. Mech. Appl. Math. 17, 319 (1964).

${ }^{14}$ E. W. Schwiderski and H. J. Lugt, "Rotating flows of von Kármán and Bödewadt," Phys. Fluids 7, 867 (1964).

${ }^{15}$ J. Brady and A. Acrivos, "Closed-cavity laminar flows at moderate Reynolds numbers," J. Fluid Mech. 115, 427 (1982).

${ }^{16}$ J. Brady and L. Durlofsky, "On rotating disk flow," J. Fluid Mech. 175, 363 (1987).

${ }^{17}$ R. J. Lingwood, "Absolute instability of the Ekman layer and related rotating flows," J. Fluid Mech. 331, 405 (1997).

${ }^{18}$ S. O. MacKerrell, "Stability of Bödewadt flow," Philos. Trans. R. Soc. London, Ser. A 363, 1181 (2005).

${ }^{19}$ R. Fernandez-Feria, "Axisymmetric instabilities of Bödewadt flow," Phys. Fluids 12, 1730 (2000).

${ }^{20}$ Ö. Savaş, "Circular waves on a stationary disk in rotating flow," Phys. Fluids 26, 3445 (1983).

${ }^{21}$ Ö. Savaş, "Stability of Bödewadt flow," J. Fluid Mech. 183, 77 (1987).

${ }^{22}$ J. M. Lopez and P. D. Weidman, "Stability of stationary endwall boundary layers during spindown," J. Fluid Mech. 326, 373 (1996).

${ }^{23} \mathrm{~J}$. M. Lopez, "Flow between a stationary and a rotating disk shrouded by a co-rotating cylinder," Phys. Fluids 8, 2605 (1996).

${ }^{24}$ J. M. Lopez and J. Shen, "An efficient spectral-projection method for the Navier-Stokes equations in cylindrical geometries I. Axisymmetric cases," J. Comput. Phys. 139, 308 (1998).

${ }^{25}$ S. Hugues and A. Randriamampianina, "An improved projection scheme applied to pseudospectral methods for the incompressible Navier Stokes equations," Int. J. Numer. Methods Fluids 28, 501 (1998).

${ }^{26}$ F. Marques, I. Mercader, O. Batiste, and J. M. Lopez, "Centrifugal effects 
in rotating convection: Axisymmetric states and three-dimensional instabilities," J. Fluid Mech. 580, 303 (2007).

${ }^{27}$ J. M. Lopez, F. Marques, I. Mercader, and O. Batiste, "Onset of convection in a moderate aspect-ratio rotating cylinder: Eckhaus-Benjamin-Feir instability," J. Fluid Mech. 590, 187 (2007).

${ }^{28}$ I. Mercader, A. Alonso, and O. Batiste, "Spatio-temporal dynamics near the onset of convection for binary mixtures in cylindrical containers," Phys. Rev. E 77, 036313 (2008).

${ }^{29}$ J. M. Lopez, "Characteristics of endwall and sidewall boundary layers in a rotating cylinder with a differentially rotating endwall," J. Fluid Mech. 359, 49 (1998).

${ }^{30} \mathrm{~N}$. Rott and W. S. Lewellen, "Boundary layers and their interactions in rotating flows," Prog. Aeronaut. Sci. 7, 111 (1966).

${ }^{31}$ G. Gauthier, P. Gondret, and M. Rabaud, "Axisymmetric propagating vortices in the flow between a stationary and a rotating disk enclosed by a cylinder," J. Fluid Mech. 386, 105 (1999).

${ }^{32}$ O. Marquet, D. Sipp, J. Chomaz, and L. Jacquin, “Amplifier and resonator dynamics of a low-Reynolds-number recirculation bubble in a global framework," J. Fluid Mech. 605, 429 (2008).

${ }^{33}$ W. Eckhaus, Studies in Nonlinear Stability Theory (Springer, Berlin, 1965).

${ }^{34}$ L. Schouveiler, P. Le Gal, and M. P. Chauve, "Stability of a traveling roll system in a rotating disk flow," Phys. Fluids 10, 2695 (1998).

${ }^{35}$ Y. Liu and R. E. Ecke, "Eckhaus-Benjamin-Feir instability in rotating convection," Phys. Rev. Lett. 78, 4391 (1997).

${ }^{36} \mathrm{D}$. Rand, "Dynamics and symmetry. predictions for modulated waves in rotating fluids," Arch. Ration. Mech. Anal. 79, 1 (1982).

${ }^{37}$ E. Serre, E. Crespo del Arco, and P. Bontoux, "Annular and spiral patterns in flows between rotating and stationary disks," J. Fluid Mech. 434, 65 (2001).
${ }^{38}$ L. Schouveiler, P. Le Gal, and M. P. Chauve, "Instabilities of the flow between a rotating and a stationary disk," J. Fluid Mech. 443, 329 (2001).

${ }^{39}$ F. Moisy, O. Doaré, T. Pasutto, O. Daube, and M. Rabaud, "Experimental and numerical study of the shear layer instability between two counterrotating disks," J. Fluid Mech. 507, 175 (2004).

${ }^{40}$ E. Serre, E. Tuliszka-Sznitko, and P. Bontoux, "Coupled numerical and theoretical study of the flow transition between a rotating and a stationary disk," Phys. Fluids 16, 688 (2004).

${ }^{41}$ A. Randriamampianina and S. Poncet, "Turbulence characteristics of the Bödewadt layer in a large enclosed rotor-stator system," Phys. Fluids 18, 055104 (2006).

${ }^{42}$ E. Séverac, S. Poncet, E. Serre, and M.-P. Chauve, "Large eddy simulation and measurements of turbulent enclosed rotor-stator flows," Phys. Fluids 19, 085113 (2007).

${ }^{43}$ J. M. Lopez, J. E. Hart, F. Marques, S. Kittelman, and J. Shen, "Instability and mode interactions in a differentially driven rotating cylinder," J. Fluid Mech. 462, 383 (2002).

${ }^{44}$ S. Poncet, E. Serre, and P. Le Gal, "Revisiting the two first instabilities of the flow in an annular rotor-stator cavity," Phys. Fluids 21, 064106 (2009).

${ }^{45}$ P. Huerre and P. A. Monkewitz, "Local and global instabilities in spatially developing flows," Annu. Rev. Fluid Mech. 22, 473 (1990).

${ }^{46}$ L. Kaiktsis, G. E. Karniadakis, and S. A. Orszag, "Unsteadiness and convective instabilities in two-dimensional flow over a backward-facing step," J. Fluid Mech. 321, 157 (1996).

${ }^{47}$ H. M. Blackburn, D. Barkley, and S. J. Sherwin, "Convective instability and transient growth in flow over a backward-facing step," J. Fluid Mech. 603, 271 (2008)

${ }^{48}$ C. Cossu and J. M. Chomaz, "Global measures of local convective instabilities," Phys. Rev. Lett. 78, 4387 (1997). 